$$
\begin{array}{lllll}
\lrcorner & \lrcorner
\end{array}
$$

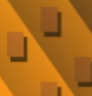

\title{
Renormalization and quantum field theory
}

Richard E. Borcherds 


\title{
Renormalization and quantum field theory
}

\author{
Richard E. Borcherds
}

The aim of this paper is to describe how to use regularization and renormalization to construct a perturbative quantum field theory from a Lagrangian. We first define renormalizations and Feynman measures, and show that although there need not exist a canonical Feynman measure, there is a canonical orbit of Feynman measures under renormalization. We then construct a perturbative quantum field theory from a Lagrangian and a Feynman measure, and show that it satisfies perturbative analogues of the Wightman axioms, extended to allow time-ordered composite operators over curved spacetimes.

\section{Introduction}

We give an overview of the construction of a perturbative quantum field theory from a Lagrangian. We start by translating some terms in physics into mathematical terminology.

Definition 1. Spacetime is a smooth finite-dimensional metrizable manifold $M$, together with a "causality" relation $\leqslant$ that is closed, reflexive, and transitive. We say that two points are spacelike separated if they are not comparable; in other words, if neither $x \leqslant y$ nor $y \leqslant x$.

The causality relation $a \leqslant b$ means informally that $a$ occurs before $b$. The causality relation will often be constructed in the usual way from a Lorentz metric with a time orientation, but since we do not use the Lorentz metric for anything else we do not bother to give $M$ one. The Lorentz metric will later appear implicitly in the choice of a cut propagator, which is often constructed using a metric.

Definition 2. The sheaf of classical fields $\Phi$ is the sheaf of smooth sections of some finite-dimensional super vector bundle over spacetime.

This research was supported by a Miller professorship and an NSF grant. I thank the referees for suggesting many improvements.

MSC2000: 22E70.

Keywords: quantum field theory, renormalization, Feynman measure, Hopf algebra, Feynman diagram. 
When the sheaf of classical fields is a supersheaf, one uses the usual conventions of superalgebra: in particular the symmetric algebras used later are understood to be symmetric algebras in the superalgebra sense, and the usual superalgebra minus signs should be inserted into formulas whenever the order of two terms is exchanged.

As usual, a global section of a sheaf of things is called a thing, so a classical field $\varphi$ is a global section of the sheaf $\Phi$ of classical fields, and so on. (A subtle point is sometimes things called classical fields in the physics literature are better thought of as sections of the dual of the sheaf of classical fields; in practice this distinction does not matter because the sheaf of classical fields usually comes with a bilinear form giving a canonical isomorphism with its dual.)

Definition 3. The sheaf of derivatives of classical fields or simple fields is the sheaf $J \Phi=\operatorname{Hom}(J, \Phi)$, where $J$ is the sheaf of jets of $M$ and the Hom is taken over the smooth functions on $M$, equal to the inverse limit of the sheaves of jets of finite order of $M$, as in [Grothendieck 1967, 16.3].

Definition 4. The sheaf of (polynomial) Lagrangians or composite fields $S J \Phi$ is the symmetric algebra of the sheaf $J \Phi$ of derivatives of classical fields.

Its sections are (polynomial) Lagrangians, in other words polynomial in fields and their derivations, so for example $\lambda \varphi^{4}+m^{2} \varphi^{2}+\varphi \partial_{i}^{2} \varphi$ is a Lagrangian, but $\sin (\varphi)$ is not.

Perturbative quantum field theories depend on the choice of a Lagrangian $L$, which is the sum of a free Lagrangian $L_{F}$ that is quadratic in the fields, and an interaction Lagrangian $L_{I} \in S J \Phi \otimes \mathbb{C} \llbracket \lambda_{1}, \ldots, \lambda_{n} \rrbracket$ whose coefficients are infinitesimal, in other words elements of a formal power series ring $\mathbb{C} \llbracket \lambda_{1}, \ldots, \lambda_{n} \rrbracket$ over the reals with constant terms 0 .

Definition 5. The sheaf of Lagrangian densities or local actions $\omega S J \Phi=\omega \otimes S J \Phi$ is the tensor product of the sheaf $S J \Phi$ of Lagrangians and the sheaf $\omega$ of smooth densities (taken over smooth functions on $M$ ).

For a smooth manifold, the (dualizing) sheaf $\omega$ of smooth densities (or smooth measures) is the tensor product of the orientation sheaf with the sheaf of differential forms of highest degree, and is noncanonically isomorphic to the sheaf of smooth functions. Densities are roughly "things that can be locally integrated". For example, if $M$ is oriented, then $\left(\lambda \varphi^{4}+m^{2} \varphi^{2}+\varphi \partial_{i}^{2} \varphi\right) d^{n} x$ is a Lagrangian density.

We use $\Gamma$ and $\Gamma_{c}$ to stand for spaces of global and compactly supported sections of a sheaf. These will usually be spaces of smooth functions (or compactly supported smooth functions) in which case they are topologized in the usual way so that their duals are compactly supported distributions (or distributions) taking values in some sheaf. 
Definition 6. A (nonlocal) action is a polynomial in local actions, in other words an element of the symmetric algebra $S \Gamma \omega S J \Phi$ of the real vector space $\Gamma \omega S J \Phi$ of local actions.

We do not complete the symmetric algebra, so expressions such as $e^{i \lambda L}$ are not in general nonlocal actions, unless we work over some base ring in which $\lambda$ is nilpotent.

We will use $*$ for complex conjugation and for the antipode of a Hopf algebra and for the adjoint of an operator and for the anti-involution of a $*$-algebra. The use of the same symbol for all of these is deliberate and indicates that they are all really special cases of a universal "adjoint" or "antipode" operation that acts on everything: whenever two of these operations are defined on something they are equal, so can all be denoted by the same symbol.

The quantum field theories we construct depend on the choice of a cut propagator $\Delta$ that is essentially the same as the 2-point Wightman distribution

$$
\Delta\left(\varphi_{1}, \varphi_{2}\right)=\int_{x, y}\left\langle 0\left|\varphi_{1}(x) \varphi_{2}(y)\right| 0\right\rangle d x d y
$$

Definition 7. A propagator $\Delta$ is a continuous bilinear map $\Gamma_{c} \omega \Phi \times \Gamma_{c} \omega \Phi \rightarrow \mathbb{C}$.

- $\Delta$ is called local if $\Delta(f, g)=\Delta(g, f)$ whenever the supports of $f$ and $g$ are spacelike separated.

- $\Delta$ is called Feynman if it is symmetric: $\Delta(f, g)=\Delta(g, f)$.

- $\Delta$ is called Hermitian if $\Delta^{*}=\Delta$, where $\Delta^{*}$ is defined by $\Delta^{*}\left(f^{*}, g^{*}\right)=$ $\Delta(g, f)^{*}$ (with a change in order of $f$ and $g$ ).

- $\Delta$ is called positive if $\Delta\left(f^{*}, f\right) \geqslant 0$ for all $f$.

- $\Delta$ is called cut if it satisfies the following "positive energy" condition: at each point $x$ of $M$ there is a partial order on the cotangent space defined by a proper closed convex cone $C_{x}$, such that if $(p, q)$ is in the wave front set of $\Delta$ at some point $(x, y) \in M^{2}$ then $p \leqslant 0$ and $q \geqslant 0$. Also, as a distribution, $\Delta$ can be written in local coordinates as a boundary value of something in the algebra generated by smooth functions and powers and logarithms of polynomials (the boundary values taken so that the wave front sets lie in the regions specified above). Moreover if $x=y$ then $p+q=0$.

A propagator can also be thought of as a complex distribution on $M \times M$ taking values in the dual of the external tensor product $J \Phi \otimes J \Phi$. In particular it has a wave front set (see [Hörmander 1990]) at each point of $M^{2}$, which is a cone in the imaginary cotangent space of that point. If $A$ and $B$ are in $\Gamma_{c} \Phi$, then $\Delta(A, B)$ is defined to be a compactly supported distribution on $M \times M$, defined by $\Delta(A, B)(f, g)=\Delta(A f, B f)$ for $f$ and $g$ in $\Gamma \omega$. 
The key point in the definition of a cut propagator is the condition on the wave front sets, which distinguishes the cut propagators from other propagators such as Feynman propagators or advanced and retarded propagators that can have more complicated wave front sets. For most common cut propagators in Minkowski space, this follows from the fact that their Fourier transforms have support in the positive cone. The condition about being expressible in terms of smooth functions and powers and logs of polynomials is a minor technical condition that is in practice satisfied by almost any reasonable example, and is used in the proof that Feynman measures exist.

If $\left(p_{1}, \ldots p_{n}\right)$ is in the imaginary cotangent space of a point of $M^{n}$, then we write $\left(p_{1}, \ldots p_{n}\right) \geqslant 0$ if $p_{j} \geqslant 0$ for all $j$, and call it positive if it is not zero.

Example 8. Over Minkowski space, most of the usual cut propagators are positive (except for ghost fields), local, and Hermitian. Most of the ideas for the proof of this can be seen for the simplest case of the propagator for massive Hermitian scalar fields. Using translation invariance, we can write $\Delta(x, y)=\Delta(x-y)$ for some distribution $\Delta$ on Minkowski spacetime. Then the Fourier transform of this in momentum space is a rotationally invariant measure supported on one of the two components of vectors with $p^{2}=m^{2}$. This propagator is positive because the measure in momentum space is positive. It satisfies the wave front set part of the cut condition because the Fourier transform has support in the positive cone, and explicit calculation shows that it can be written in terms of powers and logs of polynomials. It satisfies locality because it is invariant under rotations that preserve the direction of time, and under such rotations any space-like vector is conjugate to its negative, so $\Delta(x)=\Delta(-x)$ whenever $x$ is spacelike, in other words $\Delta(x, y)=$ $\Delta(y, x)$ whenever $x$ and $y$ are spacelike separated. The corresponding Feynman propagator is given by $1 /\left(p^{2}+m^{2}+i \varepsilon\right)$ where the $i \varepsilon$ indicates in which direction one integrates around the poles, so the cut propagator is just the residue of the Feynman propagator along one of the 2 components of the 2-sheeted hyperboloid $p^{2}=m^{2}$.

For other fields such as spinor fields in Minkowski space, the sheaf of classical fields will usually be some sort of spin bundle. The propagators can often be expressed in terms of the the propagator for a scalar field by acting on it with polynomials in momentum multiplied by Dirac's gamma matrices $\gamma^{\mu}$, for example $i\left(\gamma^{\mu} p_{\mu}+m\right) /\left(p^{2}-m^{2}\right)$. Unfortunately there are a bewildering number of different notational and sign conventions for gamma matrices.

Compactly supported actions give functions on the space $\Gamma \Phi$ of smooth fields, by integrating over spacetime $M$. A Feynman measure is a sort of analogue of Haar measure on a finite-dimensional real vector space. We can think of a Haar measure as an element of the dual of the space of continuous compactly supported 
functions. For infinite-dimensional vector spaces there are usually not enough continuous compactly supported functions, but instead we can define a measure to be an element of the dual of some other space of functions. We will think of Feynman measures as something like elements of the dual of all functions that are given by free field Gaussians times a compactly supported action. In other words a Feynman measure should assign a complex number to each compactly supported action, formally representing the integral over all fields of this action times a Gaussian $e^{i L_{F}}$, where we think of the action as a function of classical fields (or rather sections of the dual of the space of classical fields, which can usually be identified with classical fields). Moreover the Feynman measure should satisfy some sort of analogue of translation invariance.

The space $e^{i L_{F}} S \Gamma_{c} \omega S J \Phi$ is a free rank 1 module over $S \Gamma_{c} \omega S J \Phi$ generated by the basis element $e^{i L_{F}}$, which can be thought of either as a formal symbol or a formal power series. Its elements can be thought of as representing functions of classical fields that are given by a polynomial times the Gaussian $e^{i L_{F}}$, and will be the functions that the Feynman measure is defined on. The symmetric algebra $S \Gamma_{c} \omega S J \Phi$ is topologized as the direct sum of the spaces $S^{n} \Gamma_{c} \omega S J \Phi$, each of which is topologized by regarding it as a space of smooth test functions over $M^{n}$.

For the definition of a Feynman measure we need to extend the propagator $\Delta$ to a larger space as follows. We think of the propagator $\Delta$ as a map taking $\Gamma_{c} J \Phi \otimes$ $\Gamma_{c} J \Phi$ to distributions on $M \times M$. We then extend it a map from $\Gamma_{c} S J \Phi \times \Gamma_{c} S J \Phi$ to distributions on $M \times M$ by putting $\Delta\left(a_{1} \cdots a_{n}, b_{1} \cdots b_{n}\right)=\sum_{\sigma \in S_{n}} \Delta\left(a_{1}, b_{\sigma(1)}\right) \times$ $\cdots \times \Delta\left(a_{1}, b_{\sigma(n)}\right)$ where the sum is over all elements of the symmetric group $S_{n}$ (and defining it to be 0 for arguments of different degrees). Finally we extend it to a map from $S^{m} \Gamma_{c} S J \Phi \times S^{n} \Gamma_{c} S J \Phi$ to distributions on $M^{m} \times M^{n}$ using the "bicharacter" property: in other words $\Delta(A B, C)=\sum \Delta\left(A, C^{\prime}\right) \Delta\left(B, C^{\prime \prime}\right)$ where the coproduct of $C$ is $\sum C^{\prime} \otimes C^{\prime \prime}$, and similarly for $\Delta(A, B C)$.

Definition 9. A Feynman measure is a continuous linear map

$$
\omega: e^{i L_{F}} S \Gamma_{c} \omega S J \Phi \rightarrow \mathbb{C} .
$$

The Feynman measure is said to be associated with the propagator $\Delta$ if it satisfies the following conditions:

- Smoothness on the diagonal: Whenever $\left(p_{1}, \ldots, p_{n}\right)$ is in the wave front set of $\omega$ at the point $(x, \ldots, x)$ on the diagonal, then $p_{1}+\cdots+p_{n}=0$

- Nondegeneracy: there is a smooth nowhere vanishing function $g$ such that $\omega\left(e^{i L_{F}} v\right)$ is $\int_{M} g v$ for $v$ in $\Gamma_{c} \omega S^{0} J \Phi=\Gamma_{c} \omega$.

- Gaussian condition, or weak translation invariance: For $A \in S^{m} \Gamma_{c} \omega S J \Phi$, $B \in S^{n} \Gamma_{c} \omega S J \Phi$, with both sides interpreted as distributions on $M^{m+n}$, we 
have

$$
\omega(A B)=\sum \omega\left(A^{\prime}\right) \Delta\left(A^{\prime \prime}, B^{\prime \prime}\right) \omega\left(B^{\prime}\right)
$$

whenever there is no element in the support of $A$ that is $\leqslant$ some element of the support of $B$. Here $\sum A^{\prime} \otimes A^{\prime \prime} \in S \Gamma_{c} \omega S J \Phi \otimes S \Gamma_{c} S J \Phi$ is the image of $A$ under the map $S^{m} \Gamma_{c} \omega S J \Phi \rightarrow S^{m} \Gamma_{c} \omega S J \Phi \otimes S^{m} \Gamma_{c} S J \Phi$ induced by the coaction $\omega S J \Phi \rightarrow \omega S J \Phi \otimes S J \Phi$ of $S J \Phi$ on $\omega S J \Phi$, and similarly for $B$. The product on the right is a product of distributions, using the extended version of $\Delta$ defined just before this definition.

We explain what is going on in this definition. We would like to define the value of the Feynman measure to be a sum over Feynman diagrams, formed by joining up pairs of fields in all possible ways by lines, and then assigning a propagator to each line and taking the product of all propagators of a diagram. This does not work because of ultraviolet divergences: products of propagators need not be defined when points coincide. If these products were defined then they would satisfy the Gaussian condition, which then says roughly that if the vertices are divided into two disjoint subsets $a$ and $b$, then a Feynman diagram can be divided into a subdiagram with vertices $a$, a subdiagram with vertices $b$, and some lines between $a$ and $b$. The value $\omega(A B)$ of the Feynman diagram would then be the product of its value $\omega\left(A^{\prime}\right)$ on $a$, the product $\Delta\left(A^{\prime \prime}, B^{\prime \prime}\right)$ of all the propagators of lines joining $a$ and $b$, and its value $\omega\left(B^{\prime}\right)$ on $b$. The Gaussian condition need not make sense if some point of $a$ is equal to some point of $b$ because if these points are joined by a line then the corresponding propagator may have a bad singularity, but does make sense whenever all points of $a$ are not $\leq$ all points of $b$. The definition above says that a Feynman measure should at least satisfy the Gaussian condition in this case, when the product is well defined.

Unfortunately the standard notation $\omega$ for a dualizing sheaf, such as the sheaf of densities, is the same as the standard notation $\omega$ for a state in the theory of operator algebras, which the Feynman measure will be a special case of. It should be clear from the context which meaning of $\omega$ is intended.

If $\omega$ is a Feynman measure and $A \in e^{i L_{F}} S^{n} \Gamma_{c} \omega S J \Phi$ then $\omega(A)$ is a complex number, but can also be considered as the compactly supported density on $M^{n}$ taking a smooth $f$ to $\omega(A)(f)=\omega(A f)$. The integral of this density $\omega(A)$ over spacetime is just the complex number $\omega(A)$.

Since $e^{i L_{F}} S \Gamma_{c} \omega S J \Phi$ is a coalgebra (where elements of $\Gamma_{c} \omega S J \Phi$ are primitive and $e^{i L_{F}}$ is grouplike), the space of Feynman measures is an algebra, whose product is called convolution.

The nondegeneracy condition just excludes some uninteresting degenerate cases, such as the measure that is identically zero, and the function $g$ appearing in it is usually normalized to be 1 . The condition about smoothness on the diagonal 
implies that the product on the right in the Gaussian condition is defined. This is because $\omega$ has the property that if an element $\left(p_{1}, \ldots p_{n}\right)$ of the wave front set of some point is nonzero then its components cannot all be positive and cannot all be negative. This shows that the wave front sets are such that the product of distributions is defined.

If $A$ is in $e^{i L_{F}} S \Gamma_{c} \omega S J \Phi$, then $\omega(A)$ can be thought of as a Feynman integral

$$
\omega(A)=\int A(\varphi) \mathscr{D} \varphi,
$$

where $L_{F}$ is a quadratic action with cut propagator $\Delta$, and where $A$ is considered to be a function of fields $\varphi$. The integral is formally an integral over all classical fields. The Gaussian condition is a weak form of translation invariance of this measure under addition of classical fields. Formally, translation invariance is equivalent to the Gaussian condition with the condition about supports omitted and cut propagators replaced by Feynman propagators, but this is not well defined because the Feynman propagators can have such bad singularities that their products are sometimes not defined when two spacetime points coincide.

The Feynman propagator $\Delta_{F}$ of a Feynman measure $\omega$ is defined to be the restriction of $\omega$ to $\Gamma_{c} \omega \Phi \times \Gamma_{c} \omega \Phi$. It is equal to the cut propagator at "time-ordered" points $(x, y) \in M^{2}$ where $x \nless y$, but will usually differ if $x \leqslant y$. As it is symmetric, it is determined by the cut propagator except on the diagonal of $M \times M$. Unlike cut propagators, Feynman propagators may have singularities on the diagonal whose wave front sets are not contained in a proper cone, so that their products need not be defined.

Any symmetric algebra SX over a module $X$ has a natural structure of a commutative and cocommutative Hopf algebra, with the coproduct defined by making all elements of $X$ primitive (in other words, $\Delta x=x \otimes 1+1 \otimes x$ for $x \in X$ ). In other words, $\mathrm{SX}$ is the coordinate ring of a commutative affine group scheme whose points form the dual of $X$ under addition. For general results about Hopf algebras see [Abe 1980]. Similarly $S J \Phi$ is a sheaf of commutative cocommutative Hopf algebras, with a coaction on itself and the trivial coaction on $\omega$, and so has a coaction on $S \omega S J \Phi$, preserving the coproduct of $S \omega S J \Phi$. It corresponds to the sheaf of commutative affine algebraic groups whose points correspond to the sheaf $J \Phi$ under addition.

Definition 10. A renormalization is an automorphism of $S \omega S J \Phi$ preserving its coproduct and the coaction of $S J \Phi$. The group of renormalizations is called the ultraviolet group.

The justification for this rather mysterious definition is Theorem 15, which shows that renormalizations act simply transitively on the Feynman measures associated to a given local cut propagator. In other words, although there is no canonical 
Feynman measure on the space of classical fields, there is a canonical orbit of such measures under renormalization.

More generally, renormalizations are global sections of the sheaf of renormalizations (defined in the obvious way), but we will make no use of this viewpoint.

The (infinite-dimensional) ultraviolet group really ought to be called the "renormalization group", but unfortunately this name is already used for a quite different 1-dimensional group. The "renormalization group" is the group of positive real numbers, together with an action on Lagrangians by "renormalization group flow". The relation between the renormalization group and the ultraviolet group is that the renormalization group flow can be thought of as a nonabelian 1-cocycle of the renormalization group with values in the ultraviolet group, using the action of renormalizations on Lagrangians that will be constructed later.

The ultraviolet group is indirectly related to the Hopf algebras of Feynman diagrams introduced in [Kreimer 1998] and applied to renormalization in [Connes and Kreimer 2000], though this relation is not that easy to describe. First of all their Hopf algebras correspond to Lie algebras, and the ultraviolet group has a Lie algebra, and these two Lie algebras are related. There is no direct relation between Connes and Kreimer's Lie algebras and the Lie algebra of the ultraviolet group, in the sense that there seems to be no natural homomorphism in either direction. However there seems to be a sort of intermediate Lie algebra that has homomorphisms to both. This intermediate Lie algebra (or group) can be defined using Feynman diagrams decorated with smooth test functions rather than the sheaf $S \omega S J \Phi$ used here. Unfortunately all my attempts to explain the product of this Lie algebra explicitly have resulted in an almost incomprehensible combinatorial mess so complicated that it is unusable. Roughly speaking, the main differences between the ultraviolet group and the intermediate Lie algebra is that the Lie algebra of the ultraviolet group amalgamates all Feynman diagrams with the same vertices while the intermediate Lie algebra keeps track of individual Feynman diagrams, and the main difference between the intermediate Lie algebra and Kreimer's algebra is that the intermediate Lie algebra is much fatter than Kreimer's algebra because it has infinite-dimensional spaces of smooth functions in it. In some sense Kreimer's algebra could be thought of as a sort of skeleton of the intermediate Lie algebra.

All reasonable Feynman measures for a given free field theory are equivalent up to renormalization, but it is not easy to show that at least one exists. We do this by following the usual method of constructing a perturbative quantum field theory in physics. We first regularize the cut local propagator which produces a meromorphic family of Feynman measures, following Etingof [1999, pages 597-607] in using Bernstein's theorem [1972] on the analytic continuation of powers of a polynomial to construct the regularization. We then use an infinite renormalization to eliminate the poles of the regularized Feynman measure in order of their complexity. 
A quantum field theory satisfying the Wightman axioms [Streater and Wightman $2000, \S 3.1]$ is determined by its Wightman distributions, which are given by linear maps $\omega_{n}: T^{n} \Gamma_{c} \omega \Phi \rightarrow \mathbb{C}$ from the tensor powers of the space of test functions for each $n$. We will follow H. J. Borchers [1962] in combining the Wightman distributions into a Wightman functional $\omega: T \Gamma_{c} \omega \Phi \rightarrow \mathbb{C}$ on the tensor algebra $T \Gamma_{c} \omega \Phi$ of the space $\Gamma_{c} \omega \Phi$ of test functions (which is sometimes called a Borchers algebra or Borchers-Uhlmann algebra or BU-algebra). In order to accommodate composite operators we extend the algebra $T \Gamma_{c} \omega \Phi$ to the larger algebra $T \Gamma_{c} \omega S J \Phi$, and to accommodate time ordered operators we extend it further to $\operatorname{TS} \Gamma_{c} \omega S J \Phi$. In this set up it is clear how to accommodate perturbative quantum field theories: we just allow $\omega$ to take values in a space of formal power series $\mathbb{C} \llbracket \lambda \rrbracket=\mathbb{C} \llbracket \lambda_{1}, \lambda_{2}, \ldots \rrbracket$ rather than $\mathbb{C}$. For regularization $\omega$ sometimes takes values in a ring of meromorphic functions. There is one additional change we need: it turns out that the elements of $\Gamma_{c} \omega S J \Phi$ do not really represent operators on a space of physical states, but are better thought of as operators that map a space of incoming states to a space of outgoing states, and vice versa. If we identify the space of incoming states with the space of physical states, this means that only products of an even number of operators of $S \Gamma_{c} \omega S J \Phi$ act on the space of physical states. So the functional defining a quantum field theory is really defined on the subalgebra $T_{0} S \Gamma_{c} \omega S J \Phi$ of even degree elements.

So the main goal of this paper is to construct a linear map from $T_{0} S \Gamma_{c} \omega S J \Phi$ to $\mathbb{C}[\lambda]$ from a given Lagrangian, and to check that it satisfies analogues of the Wightman axioms.

The space of physical states of the quantum field theory can be reconstructed from $\omega$ as follows.

Definition 11. Let $\omega: T \rightarrow C$ be an $\mathbb{R}$-linear map between real $*$-algebras.

- $\omega$ is called Hermitian if $\omega^{*}=\omega$, where $\omega^{*}\left(a^{*}\right)=\omega(a)^{*}$

- $\omega$ is called positive if it maps positive elements to positive elements, where an element of a $*$-algebra is called positive if it is a finite sum of elements of the form $a^{*} a$.

- $\omega$ is called a state if it is positive and normalized by $\omega(1)=1$

- The left, right, or 2-sided kernel of $\omega$ is the largest left, right or 2-sided ideal closed under $*$ on which $\omega$ vanishes.

- The space of physical states of $\omega$ is the quotient of $T$ by the left kernel of $\omega$. Its sesquilinear form is $\langle a, b\rangle=\omega\left(a^{*} b\right)$, and its vacuum vector is the image of 1 .

- The algebra of physical operators of $\omega$ is the quotient of $T$ by the 2 -sided kernel of $\omega$. 
The algebra of physical operators is a $*$-algebra of operators with a left action on the physical states. If $\omega$ is positive or Hermitian then so is the sesquilinear form $\langle$,$\rangle . When \omega$ is Hermitian and positive and $C$ is the complex numbers the left kernel of $\omega$ is the set of vectors $a$ with $\omega\left(a^{*} a\right)=0$, and the definition of the space of physical states is essentially the GNS construction and is also the main step of the Wightman reconstruction theorem. In this case the completion of the space of physical states is a Hilbert space.

The maps $\omega$ we construct are defined on the real vector space $T_{0} S \Gamma_{c} \omega S J \Phi$ and will initially be $\mathbb{R}$-linear. It is often convenient to extend them to be $\mathbb{C} \llbracket \lambda \rrbracket$-linear maps defined on $T_{0} S \Gamma_{c} \omega S J \Phi \otimes \mathbb{C} \llbracket \lambda \rrbracket$, in which case the corresponding space of physical states will be a module over $\mathbb{C} \llbracket \lambda \rrbracket$ and its bilinear form will be sesquilinear over $\mathbb{C} \llbracket \lambda \rrbracket$.

The machinery of renormalization and regularization has little to do with perturbation theory or the choice of Lagrangian: instead, it is needed even for the construction of free field theories if we want to include composite operators. The payoff for all the extra work needed to construct the composite operators in a free field theory comes when we construct interacting field theories from free ones. The idea for constructing an interacting field theory from a free one is simple: we just apply a suitable automorphism (or endomorphism) of the algebra $T_{0} S \Gamma_{c} \omega S J \Phi$ to the free field state $\omega$ to get a state for an interacting field. For example, if we apply an endomorphism of the sheaf $\omega S J \Phi$ then we get the usual field theories of normal ordered products of operators, which are not regarded as all that interesting. For any Lagrangian $L$ there is an infinitesimal automorphism of $T_{0} S \Gamma_{c} \omega S J \Phi$ that just multiplies elements of $S \Gamma_{c} \omega S J \Phi$ by $i L$, which we would like to lift to an automorphism $e^{i L}$. The construction of an interacting quantum field theory from a Feynman measure $\omega$ and a Lagrangian $L$ is then given by the natural action $e^{-i L} \omega$ of the automorphism $e^{-i L}$ on the state $\omega$. The problem is that $e^{i L_{I}}$ is only defined if the interaction Lagrangian has infinitesimal coefficients, due to the fact that we only defined $\omega$ on polynomials times a Gaussian, so this construction only produces perturbative quantum field theories taking values in rings of formal power series. This is essentially the problem of lifting a Lie algebra elements $L_{I}$ to a group element $e^{i L_{I}}$, which is trivial for operators on finite-dimensional vector spaces, but a subtle and hard problem for unbounded operators such as $L_{I}$ that are not self adjoint. This construction works provided the interacting part of the Lagrangian not only has infinitesimal coefficients but also has compact support. We show that the more general case of Lagrangians without compact support can be reduced to the case of compact support up to inner automorphisms, at least on globally hyperbolic spacetimes, by showing that infrared divergences cancel.

Up to isomorphism, the quantum field theory does not depend on the choice of a Feynman measure or Lagrangian, but only on the choice of a propagator. In 
particular, the interacting quantum field theory is isomorphic to a free one. This does not mean that interacting quantum field theories are trivial, because this isomorphism does not preserve the subspace of simple operators, so if one only looks at the restriction to simple operators, as in the Wightman axioms, one no longer gets an isomorphism between free and interacting theories. The difference between interacting and free field theories is that one chooses a different set of operators to be the "simple" operators corresponding to physical fields.

The ultraviolet group also has a nonlinear action on the space of infinitesimal Lagrangians. A quantum field theory is determined by the choice of a Lagrangian and a Feynman measure, and this quantum field theory is unchanged if the Feynman measure and the Lagrangian are acted on by the same renormalization. This shows why the choice of Feynman measure is not that important: if one chooses a different Feynman measure, it is the image of the first by a unique renormalization, and by applying this renormalization to the Lagrangian one still gets the same quantum field theory.

Roughly speaking, we show that these quantum field theories $e^{i L_{I}} \omega$ satisfy the obvious generalizations of Wightman axioms whenever it is reasonable to expect them to do so. For example, we will show that locality holds by showing that the state vanishes on the "locality ideal" of Definition 32, the quantum field theory is Hermitian if we start with Hermitian cut propagators and Lagrangians, and we get a (positive) state if we start with a positive (non-ghost) cut propagator. We cannot expect to get Lorentz invariant theories in general as we are working over a curved spacetime, but if we work over Minkowski space and choose Lorentz invariant cut propagators then we get Lorentz invariant free quantum field theories. In the case of interacting theories Lorentz invariance is more subtle, even if the Lagrangian is Lorentz invariant. Lorentz invariance depends on the cancellation of infrared divergences as we have to approximate the Lorentz invariant Lagrangian by non-Lorentz-invariant Lagrangians with compact support, and we can only show that infrared divergences cancel up to inner automorphisms. This allows for the possibility that the vacuum is not Lorentz invariant, in other words Lorentz invariance may be spontaneously broken by infrared divergences, at least if the theory has massless particles. (It seems likely that if there are no massless particles then infrared divergences cancel and we recover Lorentz invariance, but I have not checked this in detail.)

In the final section we discuss anomalies. Fujikawa [1979] observed that anomalies arise from the lack of invariance of Feynman measures under a symmetry group, and we translate his observation into mathematical language.

The definitions above generalize to the relative case where spacetime is replaced by a morphism $X \rightarrow Y$, whose fibers can be thought of as spacetimes parametrized by $Y$. For example, the sheaf of densities $\omega$ is replaced by the dualizing sheaf or 
complex $\omega_{X / Y}$. We will make no serious use of this generalization, though the section on regularization could be thought of as an example of this where $Y$ is the spectrum of a ring of meromorphic functions.

\section{The ultraviolet group}

We describe the structure of the ultraviolet group, and show that it acts simply transitively on the Feynman measures associated with a given propagator.

Theorem 12. The map taking a renormalization $\rho: S \omega S J \Phi \rightarrow S \omega S J \Phi$ to its composition with the natural map $S \omega S J \Phi \rightarrow S^{1} \omega S^{0} J \Phi=\omega$ identifies renormalizations with the elements of $\operatorname{Hom}(S \omega S J \Phi, \omega)$ that vanish on $S^{0} \omega S J \Phi$ and that are isomorphisms when restricted to $\omega=S^{1} \omega S^{0} J \Phi$.

Proof. This is a variation of the dual of the fact that endomorphisms $\rho$ of a polynomial ring $R[x]$ correspond to polynomials $\rho(x)$, given by the image of the polynomial $x$ under the endomorphism $\rho$. It is easier to understand the dual result first, so suppose that $C$ is a cocommutative Hopf algebra and $\omega$ is a vector space (with $C$ acting trivially on $\omega$ ). Then the symmetric algebra $S \omega C=S(\omega \otimes C)$ is a commutative algebra acted on by $C$, and its endomorphisms (as a commutative algebra) correspond exactly to elements of $\operatorname{Hom}(\omega, S \omega C)$ because any such map lifts uniquely to a $C$-invariant map from $\omega$ to $\omega C$, which in turn lifts to a unique algebra homomorphism from $S \omega C$ to itself by the universal property of symmetric algebras. This endomorphism is invertible if and only if the map from $\omega$ to $\omega=S^{1} \omega C^{0}$ is invertible, where $C^{0}$ is the vector space generated by the identity of $C$.

To prove the theorem, we just take the dual of this result, with $C$ now given by $S J \Phi$. There is one small modification we need to make in taking the dual result: we need to add the condition that the element of $\operatorname{Hom}(S \omega C, \omega)$ vanishes on $S^{0} \omega C$ in order to get an endomorphism of $S \omega C$; this is related to the fact that endomorphisms of the polynomial ring $R[x]$ correspond to polynomials, but continuous endomorphisms of the power series ring $R \llbracket x \rrbracket$ correspond to power series with vanishing constant term.

The ultraviolet group preserves the increasing filtration $S^{\leqslant m} \omega S J \Phi$ and so has a natural decreasing filtration by the groups $G_{\geqslant n}$, consisting of the renormalizations that fix all elements of $S^{\leqslant n} \omega S J \Phi$. The group $G=G \geqslant 0$ is the inverse limit of the groups $G / G_{\geqslant n}$, and the commutator of $G_{\geqslant m}$ and $G_{\geqslant n}$ is contained in $G_{\geqslant m+n}$, so in particular $G_{\geqslant 1}$ is an inverse limit of nilpotent groups $G_{\geqslant 1} / G_{\geqslant n}$. The group $G_{\geqslant n}$ is a semidirect product $G_{\geqslant n+1} G_{n}$ of its normal subgroup $G_{\geqslant n+1}$ with the group $G_{n}$, consisting of elements represented by elements of $\operatorname{Hom}(S \omega S J \Phi, \omega)$ that are the identity on $S^{1} \omega S J \Phi$ if $n>0$, and vanish on $S^{m} \omega S J \Phi$ for $m>1, m \neq n+1$. 
Lemma 13. The group $G$ is $\ldots G_{2} G_{1} G_{0}$ in the sense that any element of $G$ can be written uniquely as an infinite product $\ldots g_{2} g_{1} g_{0}$ with $g_{i} \in G_{i}$, and conversely any such infinite product converges to an element of $G$.

Proof. The convergence of this product follows from the facts that all elements $g_{i}$ preserve any space $S \leqslant m \omega S J \Phi$, and all but a finite number act trivially on it. The fact that any element can be written uniquely as such an infinite product follows from the fact that $G / G_{\geqslant n}$ is essentially the product $G_{n-1} \ldots G_{2} G_{1} G_{0}$.

The natural map

$$
S \Gamma \omega S J \Phi \rightarrow \Gamma S \omega S J \Phi
$$

is not an isomorphism, because on the left the symmetric algebra is taken over the reals, while on the right it is essentially taken over smooth functions on $M$.

Lemma 14. The action of renormalizations on $\Gamma S \omega S J \Phi$ lifts to an action on $S \Gamma_{c} \omega S J \Phi$ that preserves the coproduct, the coaction of $\Gamma S J \Phi$, and the product of elements with disjoint support.

Proof. A renormalization is given by a linear map from $\Gamma_{c} S \omega S J \Phi$ to $\Gamma_{c} \omega$, which by composition with the map $S \Gamma_{c} \omega S J \Phi \rightarrow \Gamma S \omega S J \Phi$ and the "integration over $M$ " map $\Gamma_{c} \omega \rightarrow \mathbb{R}$ lifts to a linear map from $S \Gamma_{c} \omega S J \Phi$ to $\mathbb{R}$. This linear map has the special property that the product of any two elements with disjoint support vanishes, because it is multilinear over the ring of smooth functions. As in Theorem 12, the linear map gives an automorphism of $S \Gamma_{c} \omega S J \Phi$ preserving the coproduct and the coaction of $\Gamma S J \Phi$. As the linear map vanishes on products of disjoint support, the corresponding renormalization preserves products of elements with disjoint support.

In general, renormalizations do not preserve products of elements of $S \Gamma_{c} \omega S J \Phi$ that do not have disjoint support; the ones that do are those in the subgroup $G_{0}$.

Theorem 15. The group of complex renormalizations acts simply transitively on the Feynman measures associated with a given cut local propagator.

Proof. We first show that renormalizations $\rho$ act on Feynman measures $\omega$ associated with a given local cut propagator. We have to show that renormalizations preserve nondegeneracy, smoothness on the diagonal, and the Gaussian property. The first two of these are easy to check, because the value of $\rho(\omega)$ on any element is given by a finite sum of values of $\omega$ on other elements, so is smooth along the diagonal.

To check that renormalizations preserve the Gaussian property

$$
\omega(A B)=\sum \omega\left(A^{\prime}\right) \Delta\left(A^{\prime \prime}, B^{\prime \prime}\right) \omega\left(B^{\prime}\right)
$$


we recall that renormalizations $\rho$ preserve products with disjoint support and also commute with the coaction of $S J \Phi$. Since $A$ and $B$ have disjoint supports we have $\rho(A B)=\rho(A) \rho(B)$. Since $\rho$ commutes with the coaction of $S J \Phi$, the image of $\rho(A)$ under the coaction of $S J \Phi$ is $\sum \rho\left(A^{\prime}\right) \otimes A^{\prime \prime}$, and similarly for $B$. Combining these facts with the Gaussian property for $\rho(A) \rho(B)$ shows that

$$
\omega(\rho(A B))=\sum \omega\left(\rho\left(A^{\prime}\right)\right) \Delta\left(A^{\prime \prime}, B^{\prime \prime}\right) \omega\left(\rho\left(B^{\prime}\right)\right) ;
$$

in other words, the renormalization $\rho$ preserves the Gaussian property.

To finish the proof, we have to show that for any two normalized smooth Feynman measures $\omega$ and $\omega^{\prime}$ with the same cut local propagator, there is a unique complex renormalization $g$ taking $\omega$ to $\omega^{\prime}$. We will construct $g=\ldots g_{2} g_{1} g_{0}$ as an infinite product, with the property that $g_{n-1} \ldots g_{0} \omega$ coincides with $\omega^{\prime}$ on $e^{i L_{F}} S \leqslant n \Gamma_{c} \omega S J \Phi$. Suppose that $g_{0}, \ldots, g_{n-1}$ have already been constructed. By changing $\omega$ to $g_{n-1} \ldots g_{0} \omega$ we may as well assume that they are all 1 , and that $\omega$ and $\omega^{\prime}$ coincide on $e^{i L_{F}} S^{\leqslant n} \Gamma_{c} \omega S J \Phi$. We have to show that there is a unique $g_{n} \in G_{n}$ such that $g_{n} \omega$ and $\omega^{\prime}$ coincide on $e^{L_{F}} S^{n+1} \Gamma_{c} \omega S J \Phi$.

The difference $\omega-\omega^{\prime}$, restricted to $e^{i L_{F}} S^{n+1} \Gamma_{c} \omega S J \Phi$, is a continuous linear function on $e^{i L_{F}} S^{n+1} \Gamma_{c} \omega S J \Phi$, which we think of as a distribution. Moreover, since both $\omega$ and $\omega^{\prime}$ are determined off the diagonal by their values on elements of smaller degree by the Gaussian property, this distribution has support on the diagonal of $M^{n+1}$. Since $\omega$ and $\omega^{\prime}$ both have the property that their wave front sets on the diagonal are orthogonal to the diagonal, the same is true of their difference $\omega-\omega^{\prime}$, so the distribution is given by a map $e^{i L_{F}} S^{n+1} \Gamma_{c} \omega S J \Phi \rightarrow \omega$. By Theorem 12 this corresponds to some renormalization $g_{n} \in G_{n}$, which is the unique element of $G_{n}$ such that $g_{n} \omega$ and $\omega^{\prime}$ coincide on $e^{i L_{F}} S^{n+1} \Gamma_{c} \omega S J \Phi$.

\section{Existence of Feynman measures}

We now show (see Theorem 21) the existence of at least one Feynman measure associated to any cut local propagator, by using regularization and renormalization. Regularization means that we construct a Feynman measure over a field of meromorphic functions, which will usually have poles at the point we are interested in, and renormalization means that we eliminate these poles by acting with a suitable meromorphic renormalization.

Lemma 16. If $f_{1}, \ldots, f_{m}$ are polynomials in several variables, then there are nonzero (Bernstein-Sato) polynomials $b_{i}$ and differential operators $D_{i}$ such that

$$
b_{i}\left(s_{1}, \ldots, s_{m}\right) f_{1}(z)^{s_{1}} \ldots f_{m}(z)^{s_{m}}=D_{i}(z)\left(f_{i}(z) f_{1}(z)^{s_{1}} \ldots f_{m}(z)^{s_{m}}\right) .
$$

Proof. Bernstein's proof [1972] of this theorem for the case $m=1$ also works for any $m$ after making the obvious minor changes, such as replacing the field of 
rational functions in one variable $s_{1}$ by the field of rational functions in $m$ variables.

Corollary 17. If $f_{1}, \ldots, f_{m}$ are polynomials in several variables then for any choice of continuous branches of the multivalued functions, $f_{1}(z)^{s_{1}} \ldots f_{m}(z)^{s_{m}}$ can be analytically continued from the region where all $s_{j}$ have positive real part to a meromorphic distribution-valued function for all complex values of $s_{1}, \ldots, s_{m}$.

Proof. This follows by using the functional equation of Lemma 16 to repeatedly decrease each $s_{j}$ by 1 , just as in Bernstein's proof for the case $m=1$.

Theorem 18. Any cut local propagator $\Delta$ has a regularization, in other words a Feynman measure with values in a ring of meromorphic functions whose cut propagator at some point is $\Delta$.

Proof. The following argument is inspired by the one in [Etingof 1999]. By using a locally finite smooth partition of unity, which exists since we assume that spacetime is metrizable, we can reduce to showing that a regularization exists locally. If a local propagator is smooth, it is easy to construct a Feynman measure for it, just by defining it as a sum of products of Feynman propagators. Now suppose we have a meromorphic family of local propagators $\Delta_{d}$ depending on real numbers $d_{i}$, given in local coordinates by a finite sum of boundary values of terms of the form

$$
s(x, y) p_{1}(x, y)^{d_{1}} \ldots p_{k}(x, y)^{d_{k}} \log \left(p_{k+1}(x, y)\right) \ldots
$$

where $s$ is smooth in $x$ and $y$, and the $p_{i}$ are polynomials, and where we choose some branch of the powers and logarithms in each region where they are nonzero. In this case the Feynman measure can also be defined as a meromorphic function of $d$ for all real $d$. To prove this, we can forget about the smooth function $s$ as it is harmless, and we can eliminate the $\log$ arithmic terms by writing $\log (p)$ as $d p^{t} / d t$ at $t=0$. For any fixed number of fields with derivatives of fixed order, the corresponding distribution is defined when all variables $d_{i}$ have sufficiently large real part, because the product of the propagators is smooth enough to be defined in this case. But this distribution is given in local coordinates by the product the $d_{i}$ 'th powers of polynomials of $x$ and $y$. By Bernstein's Corollary 17 these products can be continued as a meromorphic distribution-valued function of the $d_{i}$ to all complex $d_{i}$.

This gives a Feynman measure with values in the field of meromorphic functions in several variables, and by restricting functions to the diagonal we get a Feynman measure whose value are meromorphic functions in one variable.

Example 19 (dimensional regularization). Over Minkowski space of dimension $d$, there is a variation of the construction of a meromorphic Feynman measure, which is very similar to dimensional regularization. In dimensional regularization, 
one formally varies the dimension of spacetime, to get Feynman diagrams that are meromorphic functions of the dimension of spacetime. One way to make sense out of this is to keep the dimension of spacetime fixed, but vary the propagator of the free field theory, by considering it to be a meromorphic function of a complex number $d$. The propagator for a Hermitian scalar field, considered as a distribution of $z$ in Minkowski space, can be written as a linear combination of functions of the form

$$
\frac{K_{d / 2-1}(c \sqrt{(z, z)})}{\sqrt{(z, z)}^{d / 2-1}},
$$

where $K_{v}(z)$ is a multivalued modified Bessel function of the third kind, and where we take a suitable choice of branch (depending on whether we are considering a cut or a Feynman propagator). A similar argument using Bernstein's theorem shows that this gives a Feynman measure that is analytic in $d$ for $d$ with large real part and that can be analytically continued as a meromorphic function to all complex $d$. This gives an explicit example of a meromorphic Feynman measures for the usual propagators in Minkowski space.

Theorem 20. Any meromorphic Feynman measure can be made holomorphic by acting on it with a meromorphic renormalization.

Proof. This is essentially the result that a bare quantum field theory can be made finite by an infinite renormalization. Suppose that $\omega$ is a meromorphic Feynman measure. Using the same idea as in Theorem 15 we will construct a meromorphic renormalization $g=\ldots g_{2} g_{1} g_{0}$ as an infinite product, but this time we choose $g_{n} \in$ $G_{n}$ to kill the singularities of order $n+1$. The key point is to prove that these lowest order singularities are "local", meaning that they have support on the diagonal. (In the special case of translation-invariant theories on Minkowski spacetime this becomes the usual condition that they are "polynomials in momentum", or more precisely that their Fourier transforms are essentially polynomials in momentum on the subspace with total momentum zero). The locality follows from the Gaussian property of $\omega$, which determines $\omega$ at each order in terms of smaller orders except on the diagonal. In particular if $\omega$ is nonsingular at all orders at most $n$, then the singular parts of the order $n+1$ terms all have support on the diagonal. Since the difference is smooth along the diagonal, we can find some $g_{n} \in G_{n}$ that kills off the order $n+1$ singularities, as in Theorem 15. Since renormalizations preserve the Gaussian property we can keep on repeating this indefinitely, killing off the singularities in order of their order.

The famous problem of "overlapping divergences" is that the counterterms for individual Feynman diagrams used for renormalization sometimes contain nonpolynomial (logarithmic) terms in the momentum, which bring renormalization to a halt unless they miraculously cancel when summed over all Feynman diagrams. 
This problem is avoided in the proof above because by using the ultraviolet group we only need to handle the divergences of lowest order at each step, where it is easy to see that the logarithmic terms cancel.

Theorem 21. Any cut local propagator has an associated Feynman measure.

Proof. This follows from Theorem 18, which uses regularization to show that there is a meromorphic Feynman measure, and Theorem 20 which uses renormalization to show that the poles of this can be eliminated.

\section{Subgroups of the ultraviolet group}

There are many additional desirable properties that one can impose on Feynman measures, such as being Hermitian, or Lorentz invariant, or normal ordered, and there is often a subgroup of the ultraviolet group that acts transitively on the measures with the given property. We give several examples of this.

Example 22. A Feynman measure can be normalized so that on $S^{1} \Gamma_{c} \omega S^{0} J \Phi=$ $\Gamma_{c} \omega$ its value is given by integrating over spacetime (in other words $g=1$ in Definition 9), by acting on it by a unique element of the ultraviolet group consisting of renormalizations in $G_{0}$ that are trivial on $\omega S^{>0} J \Phi$. This group can be identified with the group of nowhere vanishing smooth complex functions on spacetime. The complementary normal subgroup of the ultraviolet group consists of the renormalizations that fix all elements of $\omega S^{0} J \Phi=\omega$, and this acts simply transitively on the normalized Feynman measures. In practice almost any natural Feynman measure one constructs is normalized.

Example 23 (normal ordering). In terms of Feynman diagrams, "normal ordering" means roughly that Feynman diagrams with an edge from a vertex to itself are discarded. We say that a Feynman measure is normally ordered if it vanishes on $\Gamma_{c} \omega S^{>0} J \Phi$. Informally, $\omega S^{>0} J \Phi$ corresponds to Feynman diagrams with just one point and edges from this point to itself. We will say that a renormalization is normally ordered if it fixes all elements of $\omega S^{>0} J \Phi$. The subgroup of normally ordered renormalizations acts transitively on the normally ordered Feynman measures. The group of all renormalizations is the semidirect product of its normal subgroup $G_{>0}$ of normally-ordered renormalizations with the subgroup $G_{0}$ preserving all products. For any renormalization, there is a unique element of $G_{0}$ that takes it to a normally ordered renormalization. The Feynman measures constructed by regularization (in particular those constructed by dimensional regularization) are usually normally ordered if the spacetime has positive dimension, but are usually not for 0-dimensional spacetimes. This is because the propagators tend to contain a factor such as $(x-y)^{-2 d}$ which vanishes for large $-d$ when $x=y$, and so vanishes on Feynman diagrams with just one point for all $d$ by analytic continuation. 
So for most purposes we can restrict to normally-ordered Feynman measures and normally-ordered renormalizations, at least for spacetimes of positive dimension.

Example 24 (normalization of Feynman propagators). In general a renormalization fixes the cut propagator but can change the Feynman propagator, by adding a distribution with support on the diagonal. However there is often a canonical choice of Feynman propagator: the one with a singularity on the diagonal of smallest possible order, which will often also be a Green function for some differential operator. We can add the condition that the Feynman propagator of a Feynman measure should be this canonical choice; the subgroup of renormalizations fixing the Feynman propagator, consisting of renormalizations fixing $S^{2} \omega J \Phi$, acts simply transitively on these Feynman measures.

Example 25. [simple operators] More generally, there is a subgroup consisting of renormalizations $\rho$ such that $\rho(a B)=\rho(a) \rho(B)$ whenever $a$ is simple (involving only one field), but where $B$ is arbitrary. This stronger condition is useful because it says (roughly) that simple operators containing only one field do not get renormalized; see the discussion in Section 6. We can find a set of Feynman measures acted on simply transitively by this group by adding the condition that

$$
\omega(a B)=\sum \Delta_{F}\left(a B_{1}\right) \omega\left(B_{2}\right)
$$

whenever $a$ is simple and $\sum B_{1} \otimes B_{2}$ is the coproduct of $B$. This relation holds whenever $a$ and $B$ have disjoint supports by definition of a Feynman measure, so the extra condition says that it also holds even when they have overlapping supports. The key point is that the product of distributions above is always defined because any nonzero element of the wave front set of $\Delta_{F}$ is of the form $(p,-p)$. This would not necessarily be true if $a$ were not simple because we would get products of more than 1 Feynman propagator whose singularities might interfere with each other. In terms of Feynman diagrams, this says that vertices with just one edge are harmless: more precisely, with this normalization, adding a vertex with just one edge to a Feynman diagram has the effect of multiplying its value by the Feynman propagator of the edge. As this condition extends the Gaussian property to more Feynman diagrams, it can also be thought of as a strengthening of the translation invariance property of the Feynman measure.

Example 26 (Dyson condition). Classically, Lagrangians were called renormalizable if all their coupling constants have nonnegative mass dimension. The filtration on Lagrangian densities by mass dimension induces a similar filtration on Feynman measures and renormalizations. The Feynman measures of mass dimension $\leqslant 0$ are acted on simply transitively by the renormalizations of mass dimension $\leqslant 0$. This is useful, because the renormalizations of mass dimension at most 0 act on the spaces 
of Lagrangian densities of mass dimension at most 0 , and these often form finitedimensional spaces, at least if some other symmetry conditions such as Lorentz invariance are added. For example, in dimension 4 the density has dimension -4 , so the (Lorentz-invariant) terms of the Lagrangian density of mass dimension at most 0 are given by (Lorentz invariant) terms of the Lagrangian of mass dimension at most 4 , such as $\varphi^{4}, \varphi^{2}, \partial \varphi \partial \varphi$, and so on: the usual Lorentz-invariant even terms whose coupling constants have mass dimension at least 0 . For example, we get a three-dimensional space of theories of the form $\lambda \varphi^{4}+m \varphi^{2}+z \partial \varphi \partial \varphi$ in this way, giving the usual $\varphi^{4}$ theory in 4 dimensions.

Example 27 (boundary terms). The Feynman measures constructed in Section 3 have the property that they vanish on "boundary terms". This means that we quotient the space of local Lagrangians $\Gamma_{c} \omega S J \Phi$ by its image under the action of smooth vector fields such as $\partial / \partial x_{i}$, or in other words we replace a spaces of $n$-forms by the corresponding de Rham cohomology group. These measures are acted on simply transitively by renormalizations corresponding to maps that vanish on boundary terms. This is useful in gauge theory, because some symmetries such as the BRST symmetry are only symmetries up to boundary terms.

Example 28 (symmetry invariance). Given a group (or Lie algebra) $G$ such as a gauge group acting on the sheaf $\Phi$ of classical fields and preserving a given cut propagator, the subgroup of $G$-invariant renormalizations acts simply transitively on the $G$-invariant Feynman measures with given cut propagator. In general there need not exist any $G$-invariant Feynman measure associated with a given cut local propagator, though if there is then $G$-invariant Lagrangians lead to $G$-invariant quantum field theories. The obstructions to finding a $G$-invariant measure are cohomology classes called anomalies, and are discussed further in Section 7.

Example 29 (Lorentz invariance). An important case of invariance under symmetry is that of Poincare invariance for flat Minkowski space. In this case the spacetime $M$ is Minkowski space, the Lie algebra $G$ is that of the Poincare group of spacetime translations and Lorentz rotations, and the cut propagator is one of the standard ones for free field theories of fields of finite spin. Then dimensional regularization is invariant under $G$, so we get a Feynman measure invariant under the Poincare group, and in particular there are no anomalies for the Poincare algebra. The elements of the ultraviolet group that are Poincare invariant act simply transitively on the Feynman measures for this propagator that are Poincare invariant. If we pick any such measure, then we get a map from invariant Lagrangians to invariant quantum field theories.

Example 30 (Hermitian conditions). The group of complex renormalizations has a real form, consisting of the subgroup of (real) renormalizations. This acts simply transitively on the Hermitian Feynman measures associated with a given cut 
local propagator. The Hermitian Feynman measures (or propagators) are not the real-valued ones, but satisfy a more complicated Hermitian condition described in Definition 36.

\section{The free quantum field theory}

We extend the Feynman measure $\omega: e^{i L_{F}} S \Gamma_{c} \omega S J \Phi \rightarrow \mathbb{C}$, which is something like a measure on classical fields, to $\omega: T e^{i L_{F}} S \Gamma_{c} \omega S J \Phi \rightarrow \mathbb{C}$. This extension, restricted to the even degree subalgebra $T_{0} e^{i L_{F}} S \Gamma_{c} \omega S J \Phi$, is the free quantum field theory. We check that it satisfies analogues of the Wightman axioms.

Formulas involving coproducts can be confusing to write down and manipulate. They are much simpler for the "grouplike" elements $g$ satisfying $\Delta(g)=g \otimes g$, $\eta(g)=1$, which form a group in any cocommutative Hopf algebra. One problem is that most of the Hopf algebras we use do not have enough grouplike elements over fields: in fact for symmetric algebras the only grouplike element is the identity. However they have plenty of grouplike elements if we add some nilpotent elements to the base field, such as $\exp (\lambda a)$ for any primitive $a$ and nilpotent $\lambda$ (in characteristic 0 ). We will adopt the convention that when we talk about grouplike elements, we are tacitly allowing extensions of the base ring by nilpotent elements.

Recall that $T e^{i L_{F}} S \Gamma_{c} \omega S J \Phi$ is the tensor algebra of $e^{i L_{F}} S \Gamma_{c} \omega S J \Phi$, with the product denoted by $\otimes$ to avoid confusing it with the product of $S \Gamma_{c} S J \Phi$. We denote the identity of $S \Gamma_{c} S J \Phi$ by 1 , and the identity of $\operatorname{TS} \Gamma_{c} \omega S J \Phi$ by $1_{T}$. The involution $*$ is defined by $\left(A_{1} \otimes \cdots \otimes A_{n}\right)^{*}=A_{n}^{*} \otimes \cdots \otimes A_{1}^{*}$, and $*$ is -1 on $\Gamma_{c} \omega S J \Phi$.

Theorem 31. If $\omega: e^{i L_{F}} S \Gamma_{c} \omega S J \Phi \rightarrow C$ is a Feynman measure, there is a unique extension of $\omega$ to $T e^{i L_{F}} S \Gamma_{c} \omega S J \Phi$ such that:

- (Gaussian condition) If $A, B_{1}, \ldots, B_{m}$ are grouplike then

$$
\begin{aligned}
& e^{-i L_{F}} \omega\left(A \otimes B_{m} \otimes \cdots \otimes B_{1}\right) \\
& \quad=\sum e^{-i L_{F}} \omega(A \otimes 1 \otimes \cdots \otimes 1) \Delta\left(A, B_{m} \ldots B_{1}\right) e^{-i L_{F}} \omega\left(B_{m} \otimes \cdots \otimes B_{1}\right) .
\end{aligned}
$$

(Both sides are considered as densities, as in Definition 9.)

- $e^{-i L_{F}} \omega(A \otimes A \otimes 1 \otimes \cdots \otimes 1)=1$ for A grouplike (Cutkosky condition; see ['t Hooft 1994, Section 6]).

Proof. We first check that all the products of distributions are well defined by examining their wave front sets. All the distributions appearing have the property that their wave front sets have no positive or negative elements. This follows by induction on the complexity of an element: if all smaller elements have this property, it implies that the products defining it are well defined, and also implies that it has the same property. 
The existence and uniqueness of $\omega$ follow because the Cutkosky condition defines it on elements of the form $A \otimes 1 \otimes 1 \otimes \cdots \otimes 1$ in terms of those of the form $A \otimes 1 \otimes \cdots \otimes 1$, and the Gaussian condition then determines it on all elements.

We can also define $\omega$ directly as follows. When the propagator is sufficiently regular then the Gaussian condition means that we can write $\omega$ on $e^{i L_{F}} S \Gamma_{c} \omega S J \Phi$ as a sum over all ways of joining up the fields of an element of $e^{i L_{F}} S \Gamma_{c} \omega S J \Phi$ in pairs, where we take the propagator of each pair and multiplying these together. This is of course essentially the usual sum over Feynman diagrams. A minor difference is that we do not distinguish between "internal" vertices associated with a Lagrangian and integrated over all spacetime, and "external" vertices associated with a field and integrated over a compact set: all vertices are associated with a composite operator that may be a Lagrangian or a simple field or a more general composite operator, and all vertices are integrated over compact sets as all coefficients are assumed to have compact support.

Similarly we can define the extension of $\omega$ to $T e^{i L_{F}} S \Gamma_{c} \omega S J \Phi$ by writing the distributions defining $\omega$ as a sum over more complicated Feynman diagrams whose vertices are in addition labeled by nonnegative integers, in such a way that

- the propagators from $A_{i}$ to $A_{i}$ are Feynman propagators,

- the propagators from $A_{i}$ to $A_{j}$ for $i<j$ are cut propagators $\Delta$, with positive wave front sets on $i$ and negative wave front sets on $j$, and

- the diagram is multiplied by a factor of $(-1)^{\operatorname{deg}\left(A_{2} A_{4} A_{6} \ldots\right)}$ (in other words, we apply $*$ to $A_{2}, A_{4}, \ldots$ )

In general, if the propagator is not sufficiently regular (so that products of propagators might not be defined when some points coincide), we can construct $\omega$ by regularization and renormalization as in Section 3, which preserves the conditions defining $\omega$.

Now we show that $\omega$ satisfies the locality property of quantum field theories (operators with spacelike-separated supports commute) by showing that it vanishes on the following locality ideal.

Definition 32. We denote by $T_{0} S \Gamma_{c} \omega S J \Phi$ the subalgebra of even degree elements of $T S \Gamma_{c} \omega S J \Phi$. The locality ideal is the 2-sided ideal of $T_{0} S \Gamma_{c} \omega S J \Phi$ spanned by the coefficients of elements of the form

$\cdots \otimes Y_{1} \otimes A B D \otimes D B C \otimes X_{n} \otimes \cdots \otimes X_{1}-\cdots \otimes Y_{1} \otimes A D \otimes D C \otimes X_{n} \otimes \cdots \otimes X_{1}$

(for $A, C \in S \Gamma_{c} \omega S J \Phi$ and $B, D \in S \Gamma_{c} \omega S J \Phi \llbracket \lambda \rrbracket$ with $B, D$ grouplike) if $n$ is even and there are no points in the support of $B$ that are $\leqslant$ any points in the support of $A$ or $C$, or if $n$ is odd and there are no points in the support of $B$ that are $\geqslant$ any points in the support of $A$ or $C$. 
The algebra $T_{0} e^{i L_{F}} S \Gamma_{c} \omega S J \Phi$ and its locality ideal are defined in the same way.

Remark 33. The map $\omega$ on $T_{0} e^{i L_{F}} S \Gamma_{c} \omega S J \Phi$ depends on the choice of Feynman measure. We can define a canonical map independent of the choice of Feynman measure by taking the underlying $*$-algebra to have elements represented by pairs $(\omega, A)$ for a Gaussian measure $\omega$ and $A \in T_{0} e^{i L_{F}} S \Gamma_{c} \omega S J \Phi$, where we identify $(\omega, A)$ with $(\rho \omega, \rho A)$ for any renormalization $\rho$. The canonical state, also denoted by $\omega$, then takes an element represented by $(\omega, A)$ to $\omega(A)$.

Theorem 34. $\omega$ vanishes on the locality ideal.

Proof. We use the notation of Definition 32. We prove this for elements with $n$ even; the case $n$ odd is similar. We can assume that the propagator $\Delta$ is sufficiently regular, as we can obtain the general case from this by regularization and renormalization. We will first do the special case when $D=1$. We can assume that $B=b_{1} \ldots b_{k}$ is homogeneous of some order $k$ and write $B_{I}$ for $\prod_{j \in I} b_{j}$. If $k=0$ then the result is obvious as $B$ is constant and both sides are the same, so we can assume that $k>0$. We show that if $k>0$ then $\omega$ vanishes on

$$
\sum_{I \cup J=\{1, \ldots k\}}(-1)^{|I|} \cdots \otimes Y_{1} \otimes A B_{I} \otimes B_{J} C \otimes X_{n} \otimes \cdots \otimes X_{1}
$$

by showing that the terms cancel out in pairs. This is because if $j$ is the index for which the support of $b_{j}$ is maximal then $\omega$ has the same value on

$$
\cdots \otimes Y_{1} \otimes A B_{I} b_{j} \otimes B_{J} C \otimes X_{n} \otimes \cdots \otimes X_{1}
$$

and

$$
\cdots \otimes Y_{1} \otimes A B_{I} \otimes b_{j} B_{J} C \otimes X_{n} \otimes \cdots \otimes X_{1} .
$$

Now we do the case of general $D$. We can assume that the support of $D$ is either $\leqslant$ all points of the support of $B$ or there are no points of it that are $\leqslant$ any points in the support of $A$ or $C$. In the first case the result follows from the special case $D=1$ by replacing $A$ and $C$ by $A D$ and $C D$. In the second case it follows from 2 applications of the special case $D=1$, replacing $B$ by $D$ and $B D$, that both terms are equal to $\cdots \otimes Y_{1} \otimes A \otimes C \otimes X_{n} \otimes \cdots \otimes X_{1}$ and are therefore equal.

This proof, in the special case that $\omega$ vanishes on $B \otimes B-1 \otimes 1$ for $B$ grouplike, is more or less the proof of unitarity of the S-matrix using the "largest time equation" given in ['t Hooft 1994, Section 6]. The locality ideal is not the largest ideal on which $\omega$ vanishes, as $\omega$ also vanishes on $A \otimes 1 \otimes 1 \otimes B-A \otimes B$; in other words we can cancel pairs $1 \otimes 1$ wherever they occur.

Theorem 35. Elements of $T_{0} S \Gamma_{c} S J \Phi$ with spacelike-separated supports commute modulo the locality ideal. 
Proof. It is sufficient to prove this for grouplike degree 2 elements, as if two even degree elements have spacelike-separated supports then they are polynomials in degree 2 elements with spacelike separated supports. We will work modulo the locality ideal. Suppose that the supports of the grouplike elements $W \otimes X \otimes Z$ and $Y$ are spacelike-separated. Then applying Theorem 34 twice gives

$$
W \otimes X \otimes Y Z=W Y \otimes X Y \otimes Y Z=W Y \otimes X \otimes Z
$$

Applying this 4 times for various values of $W, X, Y$, and $Z$ shows that if $A \otimes B$ and $C \otimes D$ are grouplike and have spacelike separated supports, then

$$
\begin{aligned}
A \otimes B \otimes C \otimes D & =A C \otimes B \otimes I \otimes D=A C \otimes I \otimes I \otimes B D=A C \otimes D \otimes I \otimes B \\
& =C \otimes D \otimes A \otimes B,
\end{aligned}
$$

so $A \otimes B$ and $C \otimes D$ commute.

Now we study when the quantum field theory $\omega$ is Hermitian, and show that we can find a Hermitian quantum field theory associated to any Hermitian local cut propagator, and show that the group of real renormalizations acts transitively on them.

Definition 36. We say that a Feynman measure $\omega$ is Hermitian if its extension to $T S \Gamma_{c} \omega S J \Phi$ is Hermitian when restricted to the even subalgebra $T_{0} S \Gamma_{c} \omega S J \Phi$.

Lemma 37. If the local cut propagator $\Delta$ is Hermitian, then it has a Hermitian Feynman measure associated with it.

Proof. We can assume that the regularization of $\Delta$ is also Hermitian, by replacing it by the average of itself and its Hermitian conjugate. We can check directly that the meromorphic family of Feynman measures associated to this Hermitian regularization is Hermitian on $T_{0} S \Gamma_{c} \omega S J \Phi$ (but not on the whole of $T S \Gamma_{c} \omega S J \Phi$ ); in other words $\omega\left(A_{n} \otimes \cdots \otimes A_{1}\right)=\omega\left(A_{1}^{*} \otimes \cdots \otimes A_{n}^{*}\right)^{*}$ if $n$ is even. For example, we get a sign factor of $-1^{\operatorname{deg}\left(A_{2}\right)+\operatorname{deg}\left(A_{4}\right)+\ldots}$ in the definition of $\omega$ on the first term, a sign factor of $-1^{\operatorname{deg}\left(A_{1}\right)+\operatorname{deg}\left(A_{3}\right)+\ldots}$ form the definition of $\omega$ for the second term, whose quotient is the factor $-1^{\operatorname{deg}\left(A_{1}\right)+\operatorname{deg}\left(A_{2}\right)+\ldots}$ coming from the action of $*$ on $A_{n} \otimes$ $\cdots \otimes A_{1}$ because $n$ is even. We can then renormalize using real renormalizations to eliminate the poles, and the resulting Feynman measure will be Hermitian.

Lemma 38. If a Feynman measure $\omega$ is Hermitian and $\rho$ is a complex renormalization, then $\rho(\omega)$ is Hermitian if and only if $\rho$ is real. In particular the subgroup of (real) renormalizations acts simply transitively on the Hermitian Feynman measures associated with a given cut local propagator.

Proof. This follows from $\rho(\omega)^{*}=\rho^{*}\left(\omega^{*}\right)$, and the fact that complex renormalizations act simply transitively on Feynman measures associated with a given cut local propagator. 
Next we show that $\omega$ is a state (in other words the space of physical states is positive definite) when the cut propagator $\Delta$ is positive, by using a representation of the physical states as a space of distributions. We define the space $H_{n}$ of $n$ particle states to be the space of continuous linear maps $S^{n} \Gamma \omega \Phi \rightarrow \mathbb{C}$ (considered as compactly supported symmetric distributions on $M^{n}$ ) whose wave front sets have no positive or negative elements, with a sesquilinear form given by

$$
\langle a, b\rangle=\int_{x, y \in M^{n}} a\left(x_{1}, \ldots\right) \prod_{j} \Delta\left(x_{j}, y_{j}\right) b\left(y_{j}, \ldots\right)^{*} d x d y .
$$

This is similar to the usual definition of the inner product on the space of states of a free field theory, except that we are using distributions rather than smooth functions. We check this is well defined. To show the product of distributions in the integral is defined we need to check that no sum of nonzero elements of the wave front sets is zero, and this follows because nonzero elements of the wave front set of the product of propagators are of the form $(p, q)$ with $p>0$ and $q<0$, but $a$ and $b$ by assumption have no positive or negative elements in their wave front sets. The integral over $M^{n}$ is well defined because $a$ and $b$ have compact support.

Lemma 39. There is a map $f$ from $T_{0} S \Gamma_{c} \omega S J \Phi$ to the orthogonal direct sum $\bigoplus H_{n}$ with

$$
\omega(A B)=\left\langle f\left(A^{*}\right), f(B)\right\rangle .
$$

Proof. By Theorem 31, $\omega(A B)$ is given by

$$
\sum \omega\left(A^{\prime}\right) \Delta\left(A^{\prime \prime}, B^{\prime \prime}\right) \omega\left(B^{\prime}\right)
$$

where $\sum A^{\prime} \otimes A^{\prime \prime}$ is the image of $A$ under the coaction of $\Gamma_{c} S J \Phi$. This is equal to $\left\langle f\left(A^{*}\right), f(B)\right\rangle$ if we define $f(A)$ as follows. Suppose that

$$
A=A_{11} A_{12} \cdots \otimes A_{21} A_{22} \ldots,
$$

and let the image of $A_{j k}$ under the coaction of $\Gamma_{c} S J \Phi$ be $\sum A_{j k}^{\prime} \otimes A_{j k}^{\prime \prime}$. Then $\omega\left(A_{11}^{\prime} A_{12}^{\prime} \cdots \otimes A_{21}^{\prime} A_{22}^{\prime} \ldots\right)$ can be regarded as a distribution on $M^{n}$, where $n$ is the total number of elements $A_{j k}$. On the other hand, $A_{11}^{\prime \prime} A_{12}^{\prime \prime} \ldots A_{21}^{\prime \prime} A_{22}^{\prime \prime} \ldots$ is a function on $M^{m}$, where $m$ is the sum of the degree of the elements $A_{j k}^{\prime \prime}$, in other words the number of fields occurring in them. There is also a map from $m$ to $n$, which induces a map from $M^{n}$ to $M^{m}$, and so by push-forward of densities a map from densities on $M^{n}$ to densities on $M^{m}$. The image $f(A)$ is then given by taking the push-forward from $M^{n}$ to $M^{m}$ of the compactly supported distribution $\omega\left(A_{11}^{\prime} A_{12}^{\prime} \cdots \otimes A_{21}^{\prime} A_{22}^{\prime} \ldots\right)$ on $M^{n}$, multiplying by the function $A_{11}^{\prime \prime} A_{12}^{\prime \prime} \ldots A_{21}^{\prime \prime} A_{22}^{\prime \prime} \ldots$ on $M^{m}$, symmetrizing the result, and repeating this for each summand of $\sum A_{j k}^{\prime} \otimes A_{j k}^{\prime \prime}$. 
Corollary 40. If the cut local propagator $\Delta$ is positive, then

$$
\omega: T e^{i L_{F}} S \Gamma_{c} \omega S J \Phi \rightarrow \mathbb{C}
$$

is a state.

Proof. This follows from the previous lemma, because if $\Delta$ is positive then so is the sesquilinear form $\langle$,$\rangle on H_{n}$, and therefore $\omega\left(A^{*} A\right)=\langle f(A), f(A)\rangle \geqslant 0$.

\section{Interacting quantum field theories}

We construct the quantum field theory of a Feynman measure and a compactly supported Lagrangian, by taking the image of the free field theory $\omega$ under an automorphism $e^{i L_{I}}$ where $L_{I}$ is the interaction part of the Lagrangian. This automorphism is only well defined if the interaction Lagrangian $L_{I}$ has infinitesimal coefficients, so the interacting quantum field theories we construct are perturbative theories taking values in rings of formal power series $\mathbb{C}[\lambda]=\mathbb{C}\left[\lambda_{1}, \ldots\right]$ in the coupling constants $\lambda_{1}, \ldots$. (By "infinitesimal" we mean elements of formal power series rings with vanishing constant term.) We then lift the construction to all actions (possible without compact support) by showing that infrared divergences cancel up to inner automorphisms.

Lemma 41. The Hopf algebra $S \Gamma_{c} \omega S J \Phi$ acts on the algebra $T_{0} S \Gamma_{c} \omega S J \Phi$, and maps the locality ideal to itself. Group-like Hermitian elements of the Hopf algebra $S \Gamma_{c} \omega S J \Phi \llbracket \lambda \rrbracket$ preserve the subset of positive elements, and therefore act on the space of states of $T_{0} S \Gamma_{c} \omega S J \Phi \llbracket \lambda \rrbracket$.

Proof. Group-like elements are algebra automorphisms, and if they are also Hermitian they commute with the involution $*$. In particular grouplike Hermitian elements preserve the set of positive elements (generated by positive linear combinations of elements of the form $a^{*} a$ ), and so map positive linear forms to positive linear forms.

Definition 42. The quantum field theory of a Lagrangian $L=L_{F}+L_{I}$, where $L_{I}$ has compact support and infinitesimal coefficients, is $e^{-i L} \omega: T_{0} S \Gamma_{c} \omega S J \Phi \rightarrow$ $\mathbb{C} \llbracket \lambda \rrbracket$.

The Hopf algebra $S \Gamma_{c} \omega S J \Phi$ acts on the vector space $S \Gamma_{c} \omega S J \Phi$ by multiplication, so grouplike elements of the form $e^{i L_{F}+i L_{I}}$ take $S \Gamma_{c} \omega S J \Phi$ to $e^{i L_{F}} S \Gamma_{c} \omega S J \Phi$ and $T_{0} S \Gamma_{c} \omega S J \Phi$ to $T_{0} e^{i L_{F}} S \Gamma_{c} \omega S J \Phi$. Since $\omega$ is in the dual of $T_{0} e^{i L_{F}} S \Gamma_{c} \omega S J \Phi$, this shows that $e^{-i L} \omega$ is in the dual of $T_{0} S \Gamma_{c} \omega S J \Phi$.

Corollary 43 (locality). Elements of $T_{0} S \Gamma_{c} \omega S J \Phi$ with spacelike-separated supports commute when acting on the space of physical states of $e^{-i L} \omega$. 
Proof. By Theorem 34 the operators of the locality ideal act trivially on the space of physical states of $\omega$. Since $e^{-i L}$ preserves the locality ideal, the locality ideal also acts trivially on the space of physical states of $e^{-i L} \omega$. By Theorem 35 this implies that operators with spacelike separated supports commute on this space.

This constructs the quantum field theory of a Lagrangian whose interaction part has compact support (and is infinitesimal). We now extend this to the case when the interaction part need not have compact support. We do this by using a cutoff function to give the Lagrangian compact support, and then we then try to show that the result is independent of the choice of cutoff function, provided it is 1 in a sufficiently large region. To do this we need to assume that spacetime is globally hyperbolic, and we also find that the result is not quite independent of the choice of cutoff.

If $f$ is a smooth function on $M$ then multiplication by $f$ is a linear transformation of $\Gamma \omega S J \Phi$ and therefore induces a homomorphism of $S \Gamma \omega S J \Phi$, denoted by $A \rightarrow A^{f}$. If $A=e^{i L}$ is grouplike, then $A^{f}=e^{i L f}$. If $f$ has compact support then so does $A^{f}$ so that $A^{f} \omega$ is defined. We try to extend the definition of $A^{f} \omega$ to more general functions $f$ in the hope that we can take $f$ to be close to 1 .

Lemma 44. Suppose that $f$ and $g$ are compactly supported smooth functions on $M$ and $n$ is even. If $f=g$ on the past of $A_{1} \ldots A_{n}$ then (modulo the locality ideal)

$$
e^{-i L_{F}} A^{f} \omega\left(A_{n} \otimes \cdots \otimes A_{1}\right)=e^{-i L_{F}} A^{g} \omega\left(A_{n} \otimes \cdots \otimes A_{1}\right)
$$

If $f=g$ on the future of $A_{1} \ldots A_{n}$ then

$e^{-i L_{F}} A^{f} \omega\left(A_{n} \otimes \cdots \otimes A_{1}\right)=e^{-i L_{F}} A^{g} \omega\left(A^{g-f} \otimes 1 \otimes A_{n} \otimes \cdots \otimes A_{1} \otimes 1 \otimes A^{g-f}\right)$

Proof. We work modulo the locality ideal. The first equality follows from

$$
A^{-f} A_{n} \otimes \cdots \otimes A^{-f} A_{1}=A^{-g} A_{n} \otimes \cdots \otimes A^{-g} A_{1}
$$

which in turn follows from Theorem 34 by repeatedly inserting $A^{f-g} \otimes A^{f-g}$ (using the fact that $n$ is even). The second equality follows in the same way from

$$
\begin{aligned}
& A^{-f} \otimes A^{-f} \otimes A^{-f} A_{n} \otimes \cdots \otimes A^{-f} A_{1} \otimes A^{-f} \otimes A^{-f} \\
= & A^{-f} \otimes A^{-g} \otimes A^{-g} A_{n} \otimes \cdots \otimes A^{-g} A_{1} \otimes A^{-g} \otimes A^{-f} .
\end{aligned}
$$

This lemma shows that the restriction of $A^{f} \omega$ to arguments with support in some fixed compact subset of $M$ is almost independent of the choice of $f$ provided that $f$ is 1 on the convex hull of the argument: different choices of $f$ are related by a locally inner automorphism of $T_{0} S \Gamma_{c} \omega S J \Phi$, given by conjugation by elements of the form $1 \otimes A^{h}$. If the spacetime is globally hyperbolic in the sense that the convex hull of a compact set is contained in a compact set, then we can always find a suitable $f$ that is 1 on the convex hull $X$ of the argument, so we can construct 
the interacting quantum field theory. The result does not depend on the choice of cutoff $f$ on the future of $X$, but does depend slightly on the choice of cutoff in the past of $X$. The choice of cutoff in the past corresponds to choices of the vacuum: roughly speaking, we turn off the interaction in the distant past, which gives different vacuums. More precisely, if we have two different cutoffs $f$ and $g$ then their vacuums, which are the images of $e^{i\left(L_{F}+f L_{I}\right)}$ and $e^{i\left(L_{F}+g L_{I}\right)}$ will differ by a factor of $e^{i(f-g) L_{I}}$. This does not change the observable physics, because all these choices of cutoffs give isomorphic quantum field theories. However it does cause difficulties in constructing a Lorentz invariant theory, because the choice of cutoff in the past is not Lorentz invariant, so the vacuums are also not Lorentz invariant, or in other words Lorentz invariance may be spontaneously broken. Presumably in theories with a mass gap one can take the limit as the cutoff in the past tends to time $-\infty$ and get a Lorentz invariant vacuum, but in theories with massless particles such as QED there is an obstruction to constructing a Lorentz invariant vacuum: Lorentz invariance might be spontaneously broken by infrared divergences. This is a well known problem, which is not worth worrying about too much, because the physical universe is not globally Lorentz invariant.

The time-ordered operator $T(A)$ of an element $A \in S \Gamma_{c} \omega S J \Phi$ is defined to be $1 \otimes A$. This has the property that

$$
T\left(A_{n} \ldots A_{1}\right)=1 \otimes A_{n} \ldots A_{1}=1 \otimes A_{n} \otimes \cdots \otimes 1 \otimes A_{1}=T\left(A_{n}\right) \ldots T\left(A_{1}\right)
$$

whenever the composite fields $A_{i} \in \Gamma_{c} \omega S J \Phi$ are in order of increasing time of their supports. This formula is sometimes used as a "definition" of the time-ordered product $T\left(A_{n} \ldots A_{1}\right)$, though this does not define it when some of the factors have overlapping supports, and in general the time-ordered product depends on the choice of a Feynman measure $\omega$. The scattering matrix $S$ of the quantum field theory is $S=T\left(e^{i L_{I}}\right)=1 \otimes e^{i L_{I}}$; this is essentially the LSZ reduction formula of Lehmann, Symanzik, and Zimmermann [Lehmann et al. 1955].

We now show that if we change the Feynman measure, then we still get an isomorphic quantum field theory provided we make a suitable change in the Lagrangian. If we change $\omega$ to a different Feynman measure for the same cut local propagator, these will differ by a unique renormalization $\rho$; in other words the other Feynman measure will be $\rho \omega$. The quantum field theory $e^{-i L} \omega$ changes under this renormalization of $\omega$ by

$$
\begin{aligned}
e^{-i L} \omega\left(A_{1} \otimes \cdots\right)=\omega\left(e^{i L} A_{1} \otimes \ldots\right) & =\rho(\omega)\left(\rho\left(e^{i L} A_{1}\right) \otimes \cdots\right) \\
& =\rho\left(e^{-i L}\right) \rho(\omega)\left(\rho\left(e^{-i L}\right) \rho\left(e^{i L} A_{1}\right) \otimes \cdots\right),
\end{aligned}
$$

so the quantum field theory stays the same under renormalization by $\rho$ if we transform the Lagrangian by

$$
i L \rightarrow \log (\rho(\exp (i L))
$$


which is a nonlinear transformation because renormalizations need not commute with products or exponentiation, and change the operators $A_{n}$ by

$$
A_{n} \rightarrow \rho\left(e^{-i L}\right) \rho\left(e^{i L} A_{n}\right) \text {. }
$$

If $A_{n}$ is a simple operator and $\rho$ satisfies the condition of Example 25, then

$$
\rho\left(e^{i L} A_{n}\right)=\rho\left(e^{i L}\right) \rho\left(A_{n}\right)=\rho\left(e^{i L}\right) A_{n},
$$

so in this special case $A_{n}$ is unchanged, or in other words simple operators are not renormalized. The behavior of composite operators under renormalization can be quite complicated when expanded out in terms of fields. The usual Wightman distributions used to construct a quantum field theory use only simple operators, so the only effect of renormalization on Wightman distributions comes from the nonlinear transformation of the Lagrangian. This nonlinear transformation of Lagrangians is the usual action of renormalizations on Lagrangians used in physics texts to convert an infinite "bare" Lagrangian $L$ to a finite physical one $L_{0}$; the bare and physical Lagrangians are related by $i L_{0}=\log (\rho(\exp (i L))$, where $\rho$ is an infinite renormalization taking an infinite Feynman measure, such as the one given by dimensional regularization, to a finite one.

The orbit of a Lagrangian under this nonlinear action of the ultraviolet group is in general infinite-dimensional. It can sometimes be cut down to a finite-dimensional space as follows. As in Example 26, we cut down to the group of renormalizations of mass dimension at most 0 , which acts on the space of Lagrangians whose coupling constants all have mass dimension at least 0 . If we also add the condition that the Lagrangian is Lorentz invariant, then we sometimes get finite-dimensional spaces of Lagrangians. The point is that the classical fields themselves tend to have positive mass dimension, so if the coupling constants all have nonnegative mass dimension then the fields appearing in any term of the Lagrangian have total mass at most $d$ (canceling out the $-d$ coming from the density) which severely limits the possibilities. At one time the Lagrangians with all coupling constants of nonnegative mass dimension were called renormalizable Lagrangians, though now all Lagrangians are regarded as renormalizable in a more general sense where one allows an infinite number of terms in the Lagrangian.

\section{Gauge invariance and anomalies}

If a Lagrangian is invariant under some group, this does not imply that the quantum field theories we construct from it are also invariant, because as pointed out in [Fujikawa 1979] we also need to choose a Feynman measure and there may not be an invariant way of doing this. The obstructions to finding an invariant quantum field theory lie inside certain cohomology groups and are called anomalies. We 
show that if these anomalies vanish then we can construct invariant quantum field theories.

Suppose that a group $G$ acts on $S J \Phi$ and preserves the set of Feynman measures with given cut local propagator, and suppose that we have chosen one such Feynman measure $\omega$. In practice we often start with an action of a Lie algebra or superalgebra, such as that generated by the BRST operator, which can be turned into a group action in the usual way by working over a ring with nilpotent elements. If $g \in G$ then $g \omega$ is another Feynman measure with the same propagator, so

$$
\omega=\rho_{g} g \omega
$$

for a unique renormalization $\rho_{g}$. This defines a nonabelian 1-cocycle: $\rho_{g h}=$ $\rho_{g} g\left(\rho_{h}\right)$, where $g\left(\rho_{h}\right)=g \rho_{h} g^{-1}$. Since $\omega$ is invariant under $\rho_{g} g$, we find that

$$
\omega\left(e^{i L} A_{1}\right)=\omega\left(\rho_{g} g\left(e^{i L} A_{1}\right)\right)=\omega\left(e^{i L} e^{-i L} \rho_{g} g\left(e^{i L} A_{1}\right)\right),
$$

so that $e^{-L} \omega$ is invariant under the transformation that takes arguments $A_{1}$ to $e^{-i L} \rho_{g} g\left(e^{i L} A_{1}\right)$. This transformation fixes 1 if $e^{i L}$ is fixed by $\rho_{g} g$. If in addition $\rho_{g} g\left(e^{i L} A_{1}\right)=\rho_{g} g\left(e^{i L}\right) \rho_{g} g\left(A_{1}\right)$ (which is not automatic as $\rho_{g}$ need not preserve products) then $A_{1}$ is taken to $\rho_{g} g\left(A_{1}\right)$ by this transformation.

This shows that we really want a Lagrangian $L$ such that $e^{i L}$ is invariant under the modified action $e^{i L} \rightarrow \rho_{g} g\left(e^{i L}\right)$. This is not the same as asking for $\rho_{g} g(i L)=i L$ because $\rho_{g}$ need not preserve products (although $g$ usually does). In practice we usually have a Lagrangian $L$ with $L$ (and $e^{i L}$ ) invariant under $G$, and the problem is whether it can be modified to $L^{\prime}$ so that $e^{i L^{\prime}}$ is invariant under the twisted action. The powers of $L$ span a coalgebra all of whose elements are $G$-invariant. Conversely, given a coalgebra $C$ all of whose elements are invariant under some group action, there is a canonical $G$-invariant grouplike element associated to this coalgebra with coefficients in the dual algebra of $C$. So a fundamental question is whether the maximal coalgebra in the space of $G$-invariant classical actions is isomorphic to the maximal coalgebra in the space of actions invariant under the twisted action of $G$.

The simplest case is when one can find a $G$-invariant Feynman measure, in which case the cocycle is trivial and the twisted action of $G$ is the same as the untwisted action. In terms of the cocycle above, $\rho \omega$ is invariant for some renormalization $\omega$ if and only if $\rho_{g}=\rho^{-1} g(\rho)$ for all $g$ (where $g(\rho)=g \rho g^{-1}$ ), in other words there is an invariant measure $\omega$ if and only if the cocycle is a coboundary. This case happens, for example, when spacetime $M$ is Minkowski space and $G$ is the Lorentz or Poincare group (or one of their double covers). Dimensional regularization in this case is automatically $G$-invariant, and so gives a $G$-invariant Feynman measure. 
In the case of BRST operators, there need not be any $G$-invariant Feynman measure. In this case the following theorem shows that one can find suitable coalgebras provided that certain obstructions, called anomalies, all vanish. The renormalizations $\rho_{g}$ need not preserve products in $S \Gamma \omega S J \Phi$, but do preserve the coproduct and also fix all elements of $\Gamma \omega S J \Phi$ if they are normalized as in Example 25. So we have an action of $G$ on the space $V=\Gamma \omega S J \Phi$, which lifts to two different actions of the coalgebra SV, the first $\sigma_{1}(g)$ preserving the product, and the second $\sigma_{2}(g)=\rho_{g} \sigma_{1}(g)$ given by twisting the first by the cocycle $\rho_{g}$.

Theorem 45. Suppose that $V$ is a real vector space acted on by a group $G$, and there are two extensions $\sigma_{1} . \sigma_{2}$ of this action to the coalgebra $S V$. If the cohomology group $H^{1}(G, V)$ vanishes then the maximal coalgebras in $S V$ whose elements are fixed by these 2 actions of $G$ are isomorphic under an isomorphism fixing the elements of $V$.

Proof. We construct an isomorphism $f$ from the maximal coalgebra in the space of $\sigma_{1}$-invariant elements to the maximal coalgebra in the space of $\sigma_{2}$-invariant elements by induction on the degree of elements. We start by taking $f$ to be the identity map on elements of degree at most 1 . We can assume that the 2 actions coincide on elements of degree less than $n$, and have to find an isomorphism $f$ making them the same on elements of degree $n$, which we will do by adding elements of $V$ to a basis of the elements of degree $n$. Suppose that $a$ is an element of degree $n>1$ contained in a coalgebra of $G$-invariant elements. We want to find $v \in V$ so that

$$
\sigma_{1}(g)(a+v)=\sigma_{2}(g)(a)+v
$$

or equivalently

$$
\sigma_{1}(g)(v)-v=\sigma_{2}(g)(a)-a .
$$

The right hand side, as a function of $g$, is a 1-coboundary of an element $a \in S V$, and therefore a 1-cocycle. We show that the right hand side is in $V$. We have

$$
\Delta(a)=a \otimes 1+1 \otimes a+\sum_{i} b_{i} \otimes c_{i}
$$

for some elements $b_{i}$ and $c_{i}$ of degrees less than $n$ invariant under $G$ (for both actions, which coincide on elements of degree less than $n$ ). Applying $\sigma_{2}$ we find that $\Delta\left(\sigma_{2}(g) a\right)=\sigma_{2}(g) a \otimes 1+1 \otimes \sigma_{2}(g) a+\sum_{i} b_{i} \otimes c_{i}$, so subtracting these two identities shows that $\sigma_{2}(g)(a)-a$ is a primitive element of $\mathrm{SV}$ and therefore in $V$. Therefore the right hand side, as a function of $g$, is a 1-cocycle with values in $V$. The solvability of the condition for $v$ says exactly that this expression is the coboundary of some element $v \in V$. In other words the obstruction to finding a suitable $v$ is exactly an element of the cohomology group $H^{1}(G, V)$, so as we assume this group vanishes we can always solve for $v$. 
Example 46. We take $V$ to be $\Gamma \omega S J \Phi$, and $G$ to be some group acting on $V$. Then the spaces of classical and quantum actions are coalgebras acted on by $G$, whose primitive elements can be identified with $V$. If $H^{1}(G, \Gamma \omega S J \Phi)$ vanishes, then the maximal $G$-invariant coalgebra in the coalgebra of classical actions is isomorphic to the maximal $G$-invariant coalgebra in the coalgebra of quantum actions. So if $L$ is a $G$-invariant classical Lagrangian, then $e^{L}$ is a $G$-invariant classical action, so gives a $G$-invariant quantum action. One cannot get a $G$-invariant quantum action by exponentiating a $G$-invariant quantum Lagrangian because the space of quantum actions does not in general have a $G$-invariant product.

Example 47. Sometimes the group $G$ only fixes classical Lagrangians up to boundary terms, in other words the Lagrangian is a $G$-invariant element of $\Gamma \omega S J \Phi / D$. In this case one replaces the cohomology group $H^{1}(G, \Gamma \omega S J \Phi)$ by

$$
H^{1}(G, \Gamma \omega S J \Phi / D) \text {. }
$$

The element $e^{i L_{F}}$ lies in the completion of $S \Gamma \omega S J \Phi$ and is fixed by the zeroth order part of the BRST operator. So the BRST operator acts on $e^{i L_{F}} S \Gamma \omega S J \Phi$.

The groups $H^{1}(G, \Gamma \omega S J \Phi)$ and $H^{1}(G, \Gamma \omega S J \Phi / D)$ (and their variations for Poincare invariant Lagrangians) for the BRST operators of gauge theories have been calculated in many cases, at least for the case of Minkowski space (see [Barnich et al. 2000], for example) and are sometimes zero, in which case corresponding invariant quantum field theories exist.

\section{References}

[Abe 1980] E. Abe, Hopf algebras, Cambridge Tracts in Mathematics 74, Cambridge University Press, Cambridge, 1980. MR 83a:16010 Zbl 0476.16008

[Barnich et al. 2000] G. Barnich, F. Brandt, and M. Henneaux, "Local BRST cohomology in gauge theories”, Phys. Rep. 338:5 (2000), 439-569. MR 2002d:81208 Zbl 1097.81571

[Bernstein 1972] I. N. Bernstein, "The analytic continuation of generalized functions with respect to a parameter", Funkcional. Anal. i Priložen. 6:4 (1972), 26-40. In Russian; translated in Funct. Anal. Appl. 6 (1973), 273-285. MR 47 \#9269 Zbl 0282.46038

[Borchers 1962] H.-J. Borchers, "On structure of the algebra of field operators", Nuovo Cimento (10) 24 (1962), 214-236. MR 25 \#5713 Zbl 0129.42205

[Connes and Kreimer 2000] A. Connes and D. Kreimer, "Renormalization in quantum field theory and the Riemann-Hilbert problem, I: The Hopf algebra structure of graphs and the main theorem", Comm. Math. Phys. 210:1 (2000), 249-273. MR 2002f:81070

[Etingof 1999] P. Etingof, "Note on dimensional regularization", pp. 597-607 in Quantum fields and strings: a course for mathematicians, vol. 1, edited by P. Deligne et al., Amer. Math. Soc., Providence, RI, 1999. MR 2001k:81177 Zbl 1137.81358

[Fujikawa 1979] K. Fujikawa, "Path-integral measure for gauge-invariant fermion theories", Phys. Rev. Lett. 42:18 (1979), 1195-1198. 
[Grothendieck 1967] A. Grothendieck, "Éléments de géométrie algébrique, IV: Étude locale des schémas et des morphismes de schémas IV”, Inst. Hautes Études Sci. Publ. Math. 32 (1967), 361. MR 39 \#220

['t Hooft 1994] G. 't Hooft, Under the spell of the gauge principle, Advanced Series in Mathematical Physics 19, World Scientific, River Edge, NJ, 1994. MR 96k:81165 Zbl 0881.53063

[Hörmander 1990] L. Hörmander, The analysis of linear partial differential operators, I: Distribution theory and Fourier analysis, 2nd ed., Grundlehren der Math. Wiss. 256, Springer, Berlin, 1990. Reprinted in Classics of Mathematics series, Springer, 2003. MR 91m:35001a Zbl 0712.35001

[Kreimer 1998] D. Kreimer, "On the Hopf algebra structure of perturbative quantum field theories", Adv. Theor. Math. Phys. 2:2 (1998), 303-334. MR 99e:81156

[Lehmann et al. 1955] H. Lehmann, K. Symanzik, and W. Zimmermann, "Zur Formulierung quantisierter Feldtheorien”, Nuovo Cimento (10) 1 (1955), 205-225. MR 17,219h Zbl 0066.44006

[Streater and Wightman 2000] R. F. Streater and A. S. Wightman, PCT, spin and statistics, and all that, Princeton Landmarks in Physics, Princeton University Press, Princeton, NJ, 2000. Corrected third printing of the 1978 edition. MR 2003f:81154 Zbl 1026.81027

Communicated by Bjorn Poonen

Received 2010-08-23 Revised 2011-02-18 Accepted 2011-04-24

reb@math.berkeley.edu Department of Mathematics, University of California, Berkeley, CA 94720-3840, United States 


\section{Algebra \& Number Theory}

www.jant.org

\section{EDITORS}

MANAGING EDITOR

Bjorn Poonen

Massachusetts Institute of Technology

Cambridge, USA

\author{
EDITORIAL BOARD CHAIR \\ David Eisenbud \\ University of California \\ Berkeley, USA
}

BOARD OF EDITORS

Georgia Benkart

Dave Benson

Richard E. Borcherds

John H. Coates

J-L. Colliot-Thélène

Brian D. Conrad

Hélène Esnault

Hubert Flenner

Edward Frenkel

Andrew Granville

Joseph Gubeladze

Ehud Hrushovski

Craig Huneke

Mikhail Kapranov

Yujiro Kawamata

János Kollár

Yuri Manin

Barry Mazur

Philippe Michel

Susan Montgomery
University of Wisconsin, Madison, USA

University of Aberdeen, Scotland

University of California, Berkeley, USA

University of Cambridge, UK

CNRS, Université Paris-Sud, France

University of Michigan, USA

Universität Duisburg-Essen, Germany

Ruhr-Universität, Germany

University of California, Berkeley, USA

Université de Montréal, Canada

San Francisco State University, USA

Hebrew University, Israel

University of Kansas, USA

Yale University, USA

University of Tokyo, Japan

Princeton University, USA

Northwestern University, USA

Harvard University, USA

École Polytechnique Fédérale de Lausanne

University of Southern California, USA
Shigefumi Mori

Raman Parimala

Jonathan Pila

Victor Reiner

Karl Rubin

Peter Sarnak

Joseph H. Silverman

Michael Singer

Ronald Solomon

Vasudevan Srinivas

J. Toby Stafford

Bernd Sturmfels

Richard Taylor

Ravi Vakil

Michel van den Bergh

Marie-France Vignéras

Kei-Ichi Watanabe

Andrei Zelevinsky

Efim Zelmanov
RIMS, Kyoto University, Japan

Emory University, USA

University of Oxford, UK

University of Minnesota, USA

University of California, Irvine, USA

Princeton University, USA

Brown University, USA

North Carolina State University, USA

Ohio State University, USA

Tata Inst. of Fund. Research, India

University of Michigan, USA

University of California, Berkeley, USA

Harvard University, USA

Stanford University, USA

Hasselt University, Belgium

Université Paris VII, France

Nihon University, Japan

Northeastern University, USA

University of California, San Diego, USA

\section{PRODUCTION}

contact@msp.org

Silvio Levy, Scientific Editor

See inside back cover or www.jant.org for submission instructions.

The subscription price for 2011 is US $\$ 150 /$ year for the electronic version, and \$210/year (+ \$35 shipping outside the US) for print and electronic. Subscriptions, requests for back issues from the last three years and changes of subscribers address should be sent to Mathematical Sciences Publishers, Department of Mathematics, University of California, Berkeley, CA 94720-3840, USA.

Algebra \& Number Theory (ISSN 1937-0652) at Mathematical Sciences Publishers, Department of Mathematics, University of California, Berkeley, CA 94720-3840 is published continuously online. Periodical rate postage paid at Berkeley, CA 94704, and additional mailing offices.

ANT peer review and production are managed by EditFLOW ${ }^{\circledR}$ from Mathematical Sciences Publishers.

PUBLISHED BY

mathematical sciences publishers

http://msp.org/

A NON-PROFIT CORPORATION

Typeset in LATEX $_{\mathrm{E}}$

Copyright $\odot 2011$ by Mathematical Sciences Publishers 


\section{Algebra \& Number Theory}

\section{Volume $5 \quad$ No. $5 \quad 2011$}

Mutation classes of $\tilde{A}_{n}$-quivers and derived equivalence classification of cluster tilted algebras of type $\tilde{A}_{n}$

$$
\text { JANINE BASTIAN }
$$

Kazhdan-Lusztig polynomials and drift configurations

LI LI and ALEXANDER YONG

Renormalization and quantum field theory

RICHARD E. BORCHERDS

Density of rational points on isotrivial rational elliptic surfaces 